\title{
Reliability and Validity of SARC-F Questionnaire to Assess Sarcopenia Among Vietnamese Geriatric Patients
}

This article was published in the following Dove Press journal: Clinical Interventions in Aging

\author{
Tam Ngoc Nguyen ${ }^{1,2}$ \\ Anh Trung Nguyen ${ }^{1,2}$ \\ Long Quynh Khuong (D) 3 \\ Thanh Xuan Nguyen ${ }^{1,2}$ \\ Huong Thi Thu Nguyen ${ }^{1,2}$ \\ Thu Thi Hoai Nguyen (I) $1,2,4$ \\ Minh Van Hoang (D) ${ }^{3}$ \\ Thang Pham ${ }^{1,2}$ \\ Tu Ngoc Nguyen (1D ${ }^{5}$ \\ Huyen Thi Thanh Vu (ID) \\ 'Department of Geriatrics, Hanoi \\ Medical University, Hanoi, Vietnam; \\ ${ }^{2}$ Scientific Research Department, \\ National Geriatric Hospital, Hanoi, \\ Vietnam; ${ }^{3}$ Hanoi University of Public \\ Health, Hanoi, Vietnam; ${ }^{4}$ Dinh Tien \\ Hoang Institute of Medicine, Hanoi, \\ Vietnam; ${ }^{5}$ Westmead Applied Research \\ Centre, Faculty of Medicine and Health, \\ The University of Sydney, Sydney, NSW, \\ Australia; ${ }^{6}$ Hanoi Medical University \\ Hospital, Hanoi, Vietnam
}

Background: The SARC-F questionnaire has been developed as a rapid diagnostic test that can be used to screen for sarcopenia.

Aim: To investigate the reliability and validity of the Vietnamese version of SARC-F as a screening tool for sarcopenia in older patients in Vietnam.

Methods: A cross-sectional study was conducted in older people attending the outpatient clinics of the National Geriatric Hospital in Vietnam. Muscle mass (using dual-energy X-ray absorptiometry), handgrip strength and gait speed were assessed. SARC-F was validated against the three standard criteria for sarcopenia: the Foundation for the National Institutes of Health (FNIH), Asia Working Group for Sarcopenia (AWGS 2019) and European Working Group on Sarcopenia in Older People (EWGSOP2).

Results: There were 764 participants (mean age $71.5 \pm 8.9$ years). The Vietnamese SARC-F questionnaire had a good internal consistency (Cronbach's alpha 0.85). The prevalence of sarcopenia was $49.2 \%$ according to SARC-F and $48.3 \%, 61.1 \%$ and $52.6 \%$ according to FNIH, AWGS 2019 and EWGSOP2, respectively. The sensitivity and specificity of SARC-F in identifying sarcopenia were $67.1 \%$ and $66.7 \%$ (for $\mathrm{FINH}$ ), $66.7 \%$ and $67.1 \%$ (for AWGS 2019), and $64.9 \%$ and $68.2 \%$ (for EWGSOP2). The AUCs of SARC-F were $0.71-0.72$ against the three sarcopenia criteria.

Conclusion: The Vietnamese version of SARC-F questionnaire has acceptable diagnostic value for sarcopenia. SARC-F could be used as an initial screening for sarcopenia in hospital clinics.

Keywords: older patients, sarcopenia, SARC-F, Vietnam

\section{Introduction}

Sarcopenia is defined as a progressive and generalized loss of muscle mass and function. ${ }^{1}$ Sarcopenia is now recognized as an independent disease and has its own International Classification Diseases-10 code (ICD-10-CM: M62.84). ${ }^{2}$ The prevalence of sarcopenia in older people varied from $9.9 \%$ to $40.4 \%$, depending on definitions and study populations. ${ }^{3}$ According to a study in 4000 communitydwelling Chinese older adults, the average annual incidence of this condition is $3.1 \%$ over 4 years follow-up. ${ }^{4}$ Sarcopenia was independently associated with health adverse outcomes, such as frailty, falls and fractures, disabilities, hospitalization, and mortality. ${ }^{5-9}$

The early diagnosis of sarcopenia is important to prevent and manage this condition effectively. ${ }^{10,11}$ Sarcopenia is often recognized late as it is presumed to be a part
Correspondence: Huyen Thi Thanh Vu Department of Geriatrics, Hanoi Medical University, 0I Ton That Tung, Dong Da, Hanoi, Vietnam

Tel +84913531579

Email vuthanhhuyenII@hmu.edu.vn 
of "normal aging" where muscle mass and strength deteriorate slowly over lifetime. ${ }^{12}$ There are a number of operational definitions of sarcopenia, including the Foundation for the National Institutes of Health (FNIH), Asia Working Group for Sarcopenia (AWGS 2019) and European Working Group on Sarcopenia in Older People (EWGSOP2). ${ }^{13-15}$ All of these sarcopenia criteria require a measurement of muscle mass. The instruments to measure muscle mass, such as dual-energy X-ray absorptiometry (DXA), bio-impedance analysis (BIA), computed tomography (CT scanner) or magnetic resonance imaging (MRI) are not widely available, especially in low and middle-income countries such as Vietnam. The SARC-F has been proposed as a valuable, simple and inexpensive tool that could be used to initially screen for sarcopenia. A self-complete questionnaire SARC$F$ was developed based on cardinal features and consequences of sarcopenia and is composed of five questions. ${ }^{16}$ The questionnaire has been shown to be valid in a number of studies in Korea, Hong Kong and Turkey. ${ }^{17-19}$ Although SARC-F has the potential as a screening tool for sarcopenia in clinical settings, ${ }^{15}$ there has been no study on the application of this questionnaire in older patients in Vietnam. Therefore, this study aims to investigate the reliability and validity of the Vietnamese version of SARC-F questionnaire as a screening tool for sarcopenia among older patients in Vietnam.

\section{Methods}

\section{Study Design and Participants}

Reliability and validity of SARC-F questionnaire were assessed in a cross-sectional study. All consecutive patients aged $\geq 60$ years visiting the five general geriatric outpatient clinics at the National Geriatric Hospital in Hanoi from November 2017 to June 2019 were approached to take part in the study. The exclusion criteria were acute severe illness, pace-maker implanted, unable to complete the questionnaire and physical examination, and unable to obtain consent.

\section{Data Collection}

Data were collected from medical records, patient interviews and physical examinations. Trained interviewers collected the data from participants via faceto-face interviews. Anthropometric measurements and other physical assessments were performed by five trained nurses.
Questionnaires, Physical Performance and

\section{Anthropometric Measurements}

Data on age, educational level and living status (living with family or living alone) were collected. Comorbidities were assessed by using Charlson Comorbidity Index. ${ }^{20}$ Participants' weight were measured in standing position with minimal clothing, using an electronic scale (Electronic Body Scale TCS-200-RT). Participants' height were measured against a convenient flat wall, with barefoot and arms hanging freely at the side. Body mass index (BMI) was calculated as weight divided by height square $\left(\mathrm{kg} / \mathrm{m}^{2}\right)$. For the measurement of calf circumference $(\mathrm{CC})(\mathrm{cm})$, participants were asked to stand upright with feet slightly apart. The circumferences of the widest part of left and right calf were measured. The upper arm circumference $(\mathrm{cm})$ was measured at the widest part of two arms while the patients bend the elbow at a $90^{\circ}$ angle and flex the bicep. And the higher value of calf and upper arm circumference were used.

\section{Translation and Adaption of the SARC-F Questionnaire}

Participants self-completed the SARC-F questionnaires. The SARC-F composed of 5 components including strength, assistance in walking, rise from the chair, climb stairs and falls. ${ }^{16}$ The Vietnamese version of the SARC-F was adapted following standardized forward-backward translation procedure. Two independent geriatricians translated the English version into Vietnamese language. In Vietnam, people use the unit of "kg" instead of "pound"; therefore, question 1 "How much difficulty do you have in lifting and carrying 10 pounds?" was modified to "How much difficulty do you have in lifting and carrying $4.5 \mathrm{~kg}$ ?". An English native speaker who had no knowledge of the wording from the original English version conducted backward translation. The two translations were compared item by item and revised upon agreement among the authors and the three translators. The English and Vietnamese versions of the SARC-F are shown in Supplementary Table 1.

Each question was scored as follows: $0=$ none, $1=$ some, $2=$ a lot or unable. The total score of the SARC-F ranges from 0 to 10 points, and a score $\geq 4$ indicates sarcopenia. ${ }^{16}$

Measurement of Appendicular Skeletal Muscle Mass, Handgrip Strength, and Gait Speed

(1) Muscle mass: Appendicular skeletal muscle mass (ASM), which presents the appendicular fat-free mass minus the bone mineral content, was assessed using Dualenergy X-ray absorptiometry scans (DXA Medic DR C12, Mauguio, France). ASM (kg) was calculated as the sum of 
the lean mass of arms and legs. DXA was calibrated on a weekly basis.

(2) Muscle strength: Handgrip strength (HGS, in kg) was measured by dynamometer (Jamar TM Hydraulic Hand Dynamometer 5030 J1 made in USA). Participants were asked to sit on a chair, bend the elbow at a $90^{\circ}$ angle and do not touch the body. The participants gripped the dynamometer as much as possible with each hand, twice a hand. The highest value was used. Handgrip dynamometer was calibrated regularly to ensure reliable and accurate results of muscle strength.

(3) Physical performance: we used 4m-walking test to evaluate gait speed (GS, m/s). Patients were instructed to walk $4 \mathrm{~m}$ as "fast as possible safely". The use of walkers or canes was acceptable, if necessary. Handgrip strength and $4 \mathrm{~m}$-walking test were performed by a trained nurse.

\section{Assessment of Sarcopenia Using Different Criteria}

Three operational criteria for sarcopenia were separately applied as the "gold standards" because these are the most commonly used criteria in sarcopenia research: FNIH criteria, AWGS 2019 criteria and EWGSOP2 criteria. The details of these diagnostic criteria are described below:

(a) National Institutes of Health Sarcopenia Project (FNIH) criteria: ${ }^{13}$ sarcopenia was defined as having (1) Low muscle mass (defined as ALM/BMI $<0.789$ in men, $<0.512$ in women), and either (2) Low muscle strength (defined as HGS $<26 \mathrm{~kg}$ in men, $<16 \mathrm{~kg}$ in women) or (3) Low physical performance (defined as gait speed $\leq 0.8 \mathrm{~m} / \mathrm{s}$ ).

(b) Asian Working Group for Sarcopenia (AWGS 2019) criteria: $^{15}$ sarcopenia was defined as (1) Low muscle mass (defined as ALM/height ${ }^{2}$ $<7.0 \mathrm{~kg} / \mathrm{m}^{2}$ in men, $<5.4 \mathrm{~kg} / \mathrm{m}^{2}$ in women) and either (2) Low muscle strength (HGS $<28 \mathrm{~kg}$ in men, $<18 \mathrm{~kg}$ in women).

(c) European Working Group on Sarcopenia in Older People (EWGOP2) criteria: $^{14}$ sarcopenia was defined as (1) Low muscle mass (ALM/height ${ }^{2}$ $<7.0 \mathrm{~kg} / \mathrm{m}^{2}$ in men, $<5.5 \mathrm{~kg} / \mathrm{m}^{2}$ in women) and either (2) Low muscle strength (HGS $<27 \mathrm{~kg}$ in men, $<16 \mathrm{~kg}$ in women).

\section{Statistical Analyses}

Data were managed in Redcap and analyzed with $\mathrm{R}$ version 3.5.0. Frequencies and percentages were used to describe categorical variables, means and standard deviations to describe quantitative variables.

The internal consistency of the SARC-F was assessed by Cronbach's alpha and item to total correlation coefficients. The value of Cronbach's alpha $\geq 0.70$ indicating an acceptable level of internal consistency. ${ }^{21}$ The item-total correlation coefficients are Pearson's correlation which ranges from 0 to 1 , with the higher value indicating the better consistency.

To assess the validity of SARC-F, the FNIH, AWGS 2019, and EWGSOP2 criteria were used as the gold standards for diagnosing of sarcopenia, and thus the receiver operator curve (ROC) was applied to evaluate the evaluate sensitivity (Se), specificity (Sp), and area under the curve (AUC) of SARC-F for common cut-off points that widely used in previous studies (ie, 4). A higher AUC value indicates a better diagnostic ability of the SARC-F, with the cut-off of high (AUC $\geq 0.9$ ), moderate $(0.7 \leq \mathrm{AUC}$ $<0.9)$, and low $(0.5 \leq \mathrm{AUC}<0.7)$. $^{22}$ The accuracy measures the proportion of correct classifications over the total number of classifications. The positive predictive value (PPV) is the probability of having sarcopenia defined by the FNIH/AWGS 2019/EWGSOP2 in participants with $\mathrm{SARC}-\mathrm{F} \geq 4$ (true positive). The negative predictive value (NPV) is the probability of not having sarcopenia (defined by the FNIH/AWGS 2019/EWGSOP2) in participants with SARC-F $<4$ (true negative) ${ }^{23}$ The higher values of accuracy, PPV and NPV indicate the higher diagnostic validity of SARC-F.

\section{Ethics}

The study was approved by the National Geriatric Hospital Ethics Committee, Hanoi, Vietnam (No.1235/QDBVLKTW November 15, 2017). All procedures followed were in accordance with the ethical standards of the responsible committee on human experimentation and with the Helsinki Declaration of 1964, as revised in 2013. Written informed consent was obtained from participants before starting the study.

\section{Results}

During the study period, 916 were approached, of whom $802(87.6 \%)$ agreed to take part in the study. Due to missing data on physical examination or DXA measurement, 38 participants were excluded. Thus, the final study population comprised of 764 participants. 


\section{Characteristics of the Study Population}

The mean age was $71.5 \pm 8.9$ years, female accounted for $61.8 \%$. Table 1 shows the study participants' characteristics by gender. Charlson comorbidity score was $1.5 \pm 1.2$ on average.

\section{The Prevalence of Sarcopenia}

Using the FNIH criteria, the percentage of sarcopenia was $48.3 \%$ overall, $68.5 \%$ in male and $35.8 \%$ in female. The prevalence of sarcopenia was $61.1 \%(71.9 \%$ in male and $54.5 \%$ in female) based on AWGS 2019 and was 52.6\% $(69.9 \%$ in male and $42.0 \%$ in female) based on EWGSOP2. Based on the SARC-F questionnaire, $49.2 \%$ were defined as having sarcopenia $(43.5 \%$ in male and $52.8 \%$ in female) (Figure 1).

\section{Reliability}

Table 2 shows the internal consistency of the Vietnamese SARC-F questionnaire, with the Cronbach's alpha value 0.85. All of the five items were correlated to the total score of the questionnaire. Item-total correlation was in range from 0.50 to 0.86 .

\section{Validity: Comparison of SARC-F Against Different Gold Standards}

The diagnostic values of SARC-F were assessed by comparing to three operational criteria (Table 3). The accuracy of SARC-F was $66.9 \%$ against FNIH criteria, 65.8\% against AWGS 2019 criteria and 66.5\% against
EWGSOP2 criteria. The sensitivity and specificity were $67.1 \%$ and $66.7 \%$ (using FINH), $62.3 \%$ and $71.4 \%$ (using AWGS 2019), 64.9\% and 68.2\% (using EWGSOP2). The negative predictive value of SARC-F questionnaire was ranged from $55 \%$ to $68 \%$ against three criteria.

The ROC for the performance of SARC-F as a screening tool is showed in Figure 2. The AUC was higher than 0.7 (AUC $0.71,95 \%$ CI: $0.68-0.75$ based on FNIH criteria; AUC 0.71, 95\% CI: $0.67-0.74$ based on AWGS 2019 criteria, AUC 0.72 , 95\% CI: $0.68-0.75$ based on EWGSOP2 criteria).

\section{Discussion}

In this study in 764 participants attending the geriatric clinics, the Vietnamese version of the SARC-F showed good internal consistency and good validity against the FNIH, AWGS 2019 and EWGSOP2 criteria. In 2018, the International Clinical Practice Guidelines for Sarcopenia (ICFSR) highlighted that SARC-F questionnaire is well recognized as one of the best screening tools for sarcopenia in daily practice. ${ }^{24}$ In line with the previous studies, the Vietnamese SARC-F proved internally consistent. ${ }^{25}$ Malmstrom et al revealed high reliability values from cohort studies $(0.81,0.78$ and 0.76 in the African American Health study, Baltimore Longitudinal Study of Aging, and National Health and Nutrition Examination Survey, respectively). ${ }^{25}$

In terms of clinical validation of the Vietnamese SARC-F against reference definitions of sarcopenia, SARC-F had an acceptable sensitivity (62\% to $67 \%)$ and specificity (67\% to

Table I Participants' Characteristics

\begin{tabular}{|c|c|c|c|c|}
\hline Characteristics & $\begin{array}{l}\text { Total } \\
(\mathrm{n}=764)\end{array}$ & $\begin{array}{l}\text { Male } \\
(n=292)\end{array}$ & $\begin{array}{l}\text { Female } \\
(n=472)\end{array}$ & $P$ value \\
\hline Age & 71.5 (8.9) & $71.4(9.0)$ & $71.6(8.8)$ & 0.780 \\
\hline Low educational level (six years or less) & $201(26.3)$ & $62(30.8)$ & $139(69.2)$ & 0.012 \\
\hline \multicolumn{5}{|l|}{ Living with } \\
\hline Family & $720(94.2)$ & $281(39.0)$ & $439(61.0)$ & 0.063 \\
\hline Alone & $44(5.8)$ & II (25.0) & $33(75.0)$ & \\
\hline Charlson Comorbidity Index & $\mathrm{I} .5(\mathrm{I} .2)$ & $1.8(1.2)$ & $\mathrm{I} .4(\mathrm{I} .2)$ & $<0.001$ \\
\hline BMI $\left(\mathrm{kg} / \mathrm{m}^{2}\right)$ & $21.7(3.4)$ & $20.9(3.2)$ & $22.1(3.4)$ & $<0.001$ \\
\hline ASM $(k g)$ & $11.2(3.0)$ & $12.0(3.0)$ & $10.8(2.9)$ & $<0.001$ \\
\hline ASM/BMI & $0.5(0.1)$ & $0.6(0.1)$ & $0.5(0.1)$ & $<0.001$ \\
\hline ASM/Height ${ }^{2}\left(\mathrm{~kg} / \mathrm{m}^{2}\right)$ & $4.7(1.1)$ & $4.8(1.0)$ & $4.7(1.2)$ & 0.372 \\
\hline Upper arm circumferences $(\mathrm{cm})$ & $23.6(8.0)$ & $21.8(8.7)$ & $24.7(7.3)$ & $<0.001$ \\
\hline Calf circumferences $(\mathrm{cm})$ & $28.2(7.7)$ & $27.4(8.6)$ & $28.8(7.1)$ & 0.021 \\
\hline Hand grip strength (kg) & $17.4(7.8)$ & $21.3(9.3)$ & $14.9(5.6)$ & $<0.001$ \\
\hline Walking speed (m/s) & $0.6(0.3)$ & $0.7(0.3)$ & $0.6(0.3)$ & 0.017 \\
\hline
\end{tabular}

Notes: Continuous data are presented as mean \pm standard deviation. Categorical data are shown as $\mathrm{n}(\%)$

Abbreviations: BMI, body mass index; ASM, appendicular skeletal muscle. 


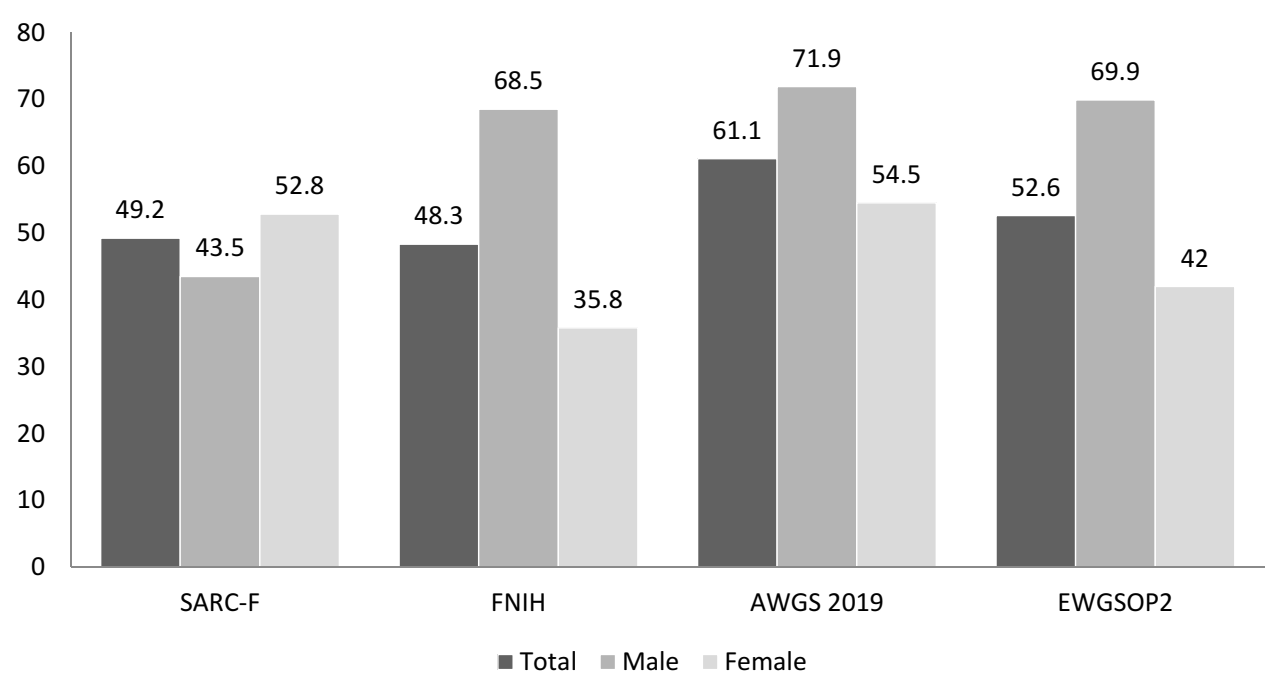

Figure I Prevalence of sarcopenia according to different criteria (SARC-F, FNIH, AWGS 2019, and EWGSOP2).

$71 \%$ ) against three operational criteria developed by European, American, and Asian consensus panels. A negative predictive value was ranging from $55 \%$ to $68 \%$. Several studies have examined the sarcopenia diagnostic value of SARC-F questionnaire among older people. ${ }^{17,18,25-28}$ In community settings, SARC-F has been shown to have high negative predictive value ( $81.6 \%$ to $98.4 \%$ ), excellent specificity ( $85 \%$ to $99 \%$ ) but poor sensitivity (3.8\% to $35.6 \%$ ). ${ }^{17,19,28}$ However, our results are similar to a study of Ida et al which found that SARC-F has higher specificity (men $85.8 \%$, women $72.4 \%$ ), lower sensitivity (men 14.6\%, women 33.3\%), and lower negative predictive value (men $67.5 \%$, women $86.2 \%$ ) in diabetic outpatients. ${ }^{27}$ The discrepancies between findings in community setting studies and hospital-based studies could be due to the fact that the prevalence of sarcopenia was much higher in older patients in clinic settings (14.3\% to $28.4 \%$ in the Ida et al diabetic patients study, and $48.9 \%$ to $61.9 \%$ in our study) compared to studies of participants recruited from the community ( $4 \%$ to $13 \%$ ). Higher pre-test probability of disease results in lower negative predictive value of diagnostic tests. ${ }^{29}$
Sarcopenia has negative impact on health outcomes and increased financial burdens when patients were diagnosed late and untreated. ${ }^{8,14}$ The implementation of the findings from sarcopenia research into health policy and action plans for clinical practice is a challenge. In our study, the diagnostic value of SARC-F questionnaire was consistent for the three operational criteria considered the "gold standard" for diagnosing sarcopenia. The result adds to evidence that SARC-F is an acceptable sarcopenic screening tool for older people in the clinical setting in Vietnam. In busy outpatient clinics, SARC-F appeared to be a good and feasible sarcopenia screening tool that could be easily used. Using SARC-F questionnaire is recommended by EWGSOP2, ICFSR and AWGS 2019 as the first step to find individuals with probable sarcopenia in community and clinical healthcare settings. ${ }^{14,15,24}$ It can help reduce the number of patients and staff exposed to radiation when measuring muscle mass by DXA, as well as the need for complicated and expensive device measurements in initial detection of sarcopenia.

Table 2 Internal Reliability of the Vietnamese SARC-F Questionnaire

\begin{tabular}{|c|c|c|c|c|c|}
\hline \multirow[t]{2}{*}{ Domains } & \multicolumn{3}{|c|}{ Score $[n(\%)]$} & \multirow[t]{2}{*}{ Mean (SD) } & \multirow{2}{*}{$\begin{array}{l}\text { Item-Total } \\
\text { Correlation }\end{array}$} \\
\hline & 0 & I & 2 & & \\
\hline Strength & $242(31.7)$ & $343(44.9)$ & $179(23.4)$ & $0.9(0.7)$ & 0.84 \\
\hline Assistance in walking & $357(46.7)$ & $380(49.7)$ & $27(3.5)$ & $0.6(0.6)$ & 0.86 \\
\hline Rise from a chair & $320(4 I .9)$ & $419(54.8)$ & $25(3.3)$ & $0.6(0.6)$ & 0.86 \\
\hline Climb stairs & $244(31.9)$ & $413(54.1)$ & $107(14.0)$ & $0.8(0.7)$ & 0.85 \\
\hline Fall history & $576(75.4)$ & $168(22.0)$ & $20(2.6)$ & $0.3(0.5)$ & 0.50 \\
\hline Total score & & & & $3.2(2.4)$ & \\
\hline
\end{tabular}


Table 3 Criterion Validity Between SARC-F and Different Sarcopenia Definitions

\begin{tabular}{|c|c|c|c|c|c|c|c|}
\hline \multirow[t]{2}{*}{ SARC-F } & $\begin{array}{l}n \\
\text { (\%) }\end{array}$ & $\begin{array}{l}n \\
\text { (\%) }\end{array}$ & \multirow[t]{2}{*}{$\begin{array}{l}\text { Acc } \\
\text { (\%) }\end{array}$} & \multirow[t]{2}{*}{$\begin{array}{l}\text { Sen } \\
\text { (\%) }\end{array}$} & \multirow[t]{2}{*}{$\begin{array}{l}\text { Spe } \\
\text { (\%) }\end{array}$} & \multirow[t]{2}{*}{$\begin{array}{l}\text { PPV } \\
\text { (\%) }\end{array}$} & \multirow[t]{2}{*}{$\begin{array}{l}\text { NPV } \\
\text { (\%) }\end{array}$} \\
\hline & $<4$ & $\geq 4$ & & & & & \\
\hline \multicolumn{8}{|l|}{ FNIH } \\
\hline No sarcopenia & $265(67.1)$ & 130 (32.9) & 66.9 & 66.7 & 67.1 & 65.4 & 68.3 \\
\hline Sarcopenia & $123(33.3)$ & $246(66.7)$ & & & & & \\
\hline \multicolumn{8}{|l|}{ AWGS 2019} \\
\hline No sarcopenia & & $\begin{array}{l}85 \\
(28.6)\end{array}$ & 65.8 & 62.3 & 71.4 & 77.4 & 54.6 \\
\hline Sarcopenia & I76 (37.7) & $291(62.3)$ & & & & & \\
\hline \multicolumn{8}{|l|}{ EWGSOP2 } \\
\hline No sarcopenia & 247 (68.2) & $115(31.8)$ & 66.5 & 64.9 & 68.2 & 69.4 & 63.7 \\
\hline Sarcopenia & $|4|(35.1)$ & $26 \mid(64.9)$ & & & & & \\
\hline
\end{tabular}

Abbreviations: Acc, accuracy; Sen, sensitivity; Spe, specificity; PPV, positive predictive value; NPV, negative predictive value.

The strengths of the study are that it investigated the reliability and validity of Vietnamese version of SARC-F questionnaire in a large sample. Muscle mass was measured using DXA, which has been approved as a part of sarcopenia diagnosis (ICD-10) and was endorsed by international working groups. ${ }^{24}$ The fast gait speeds of participants, which is a predictor of disability, were safely assessed. ${ }^{30}$ The Vietnamese version of SARC-F was appropriately translated. However, we conducted the study in a geriatric hospital, where the prevalence of sarcopenia is likely to be higher than in the community. Therefore, it is necessary to conducted further study among older adults in community settings.

\section{Conclusion}

The Vietnamese version of SARC-F questionnaire has acceptable sarcopenia diagnostic value. Our finding suggested that SARC-F could be used in initial screening for sarcopenia in hospital clinic settings in Vietnam. Further

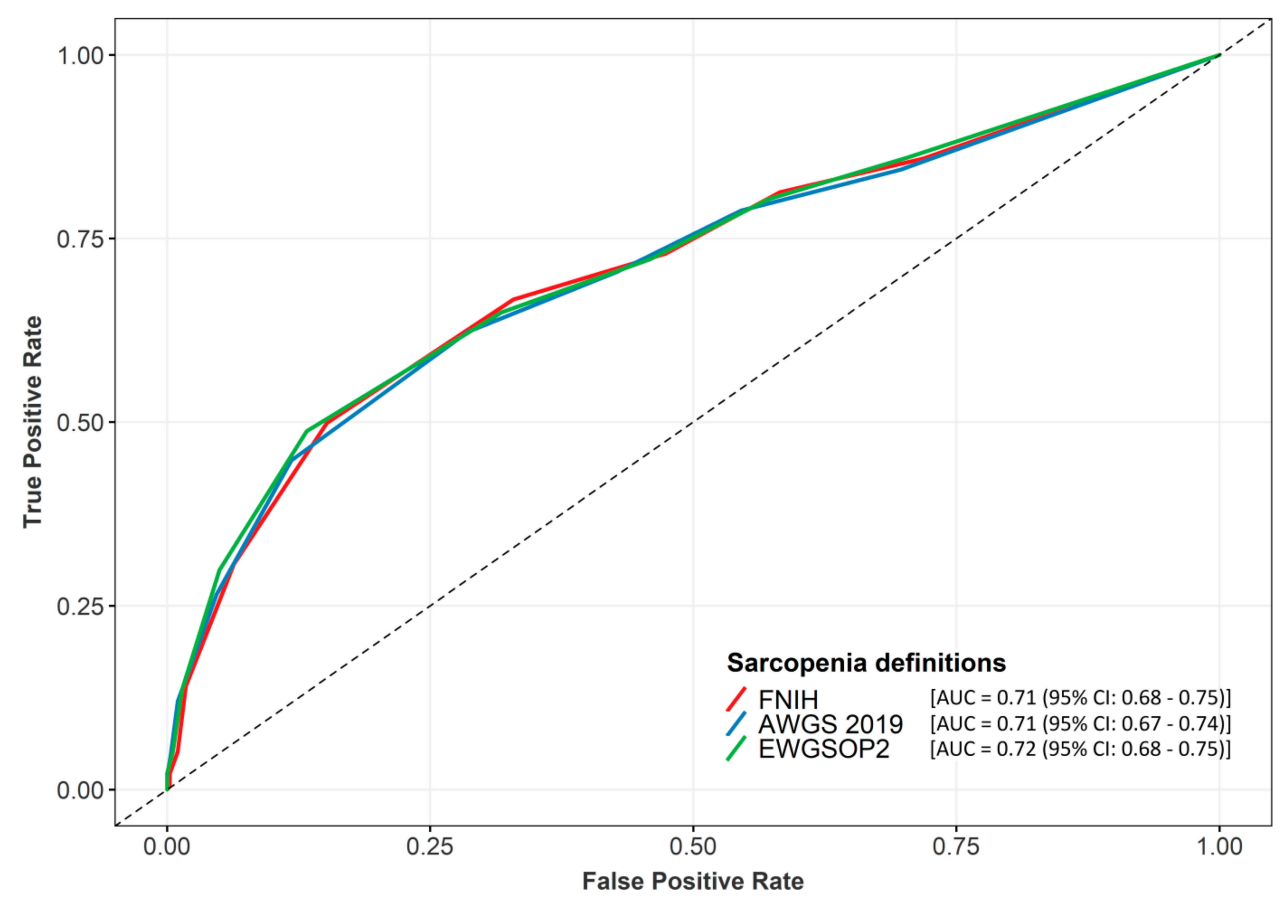

Figure 2 Receiver operator curve (ROC) of SARC-F for sarcopenia based on different sarcopenia definitions. 
longitudinal research is necessary to examine the validity of this simple tool in identification for those at risk of sarcopenia-related outcomes in Vietnam.

\section{Data Statement}

The data that support the findings of this study are available from the corresponding author upon reasonable request.

\section{Acknowledgments}

We are deeply grateful to all participants who gave their time to participate in this study. We thank Mrs. Nguyen Lan Anh for the support with inputting of the data.

\section{Author Contributions}

All authors made substantial contributions to conception and design, acquisition of data, or analysis and interpretation of data; took part in drafting the article or revising it critically for important intellectual content; gave final approval of the version to be published; and agree to be accountable for all aspects of the work.

\section{Disclosure}

The authors report no conflicts of interest in this work.

\section{References}

1. Morley JE, Baumgartner RN, Roubenoff R, Mayer J, Nair KS. Sarcopenia. J Lab Clin Med. 2001;137(4):231-243. doi:10.1067/ mlc.2001.113504

2. Anker SD, Morley JE, von Haehling S. Welcome to the ICD-10 code for sarcopenia. J Cachexia Sarcopenia Muscle. 2016;7(5):512-514. doi:10.1002/jcsm.12147

3. Mayhew A, Amog K, Phillips S, et al. The prevalence of sarcopenia in community-dwelling older adults, an exploration of differences between studies and within definitions: a systematic review and meta-analyses. Age Ageing. 2019;48(1):48-56. doi:10.1093/ageing/afy106

4. Yu R, Wong M, Leung J, Lee J, Auyeung TW, Woo J. Incidence, reversibility, risk factors and the protective effect of high body mass index against sarcopenia in community-dwelling older Chinese adults. Geriatr Gerontol Int. 2014;14:15-28. doi:10.1111/ggi.12220

5. Landi F, Liperoti R, Fusco D, et al. Sarcopenia and mortality among older nursing home residents. J Am Med Dir Assoc. 2012;13 (2):121-126. doi:10.1016/j.jamda.2011.07.004

6. Landi F, Liperoti R, Russo A, et al. Sarcopenia as a risk factor for falls in elderly individuals: results from the ilSIRENTE study. Clin nutr. 2012;31(5):652-658. doi:10.1016/j.clnu.2012.02.007

7. Yang M, Hu X, Wang H, Zhang L, Hao Q, Dong B. Sarcopenia predicts readmission and mortality in elderly patients in acute care wards: a prospective study. J Cachexia Sarcopenia Muscle. 2017;8 (2):251-258. doi:10.1002/jcsm.12163

8. Beaudart $\mathrm{C}$, Zaaria $\mathrm{M}$, Pasleau $\mathrm{F}$, Reginster J-Y, Bruyère $\mathrm{O}$. Health outcomes of sarcopenia: a systematic review and meta-analysis. PLoS One. 2017;12(1):e0169548. doi:10.1371/journal.pone.0169548

9. Han A, Bokshan SL, Marcaccio SE, DePasse JM, Daniels AH. Diagnostic criteria and clinical outcomes in sarcopenia research: a literature review. J Clin Med. 2018;7(4):70. doi:10.3390/jcm7040070
10. Roubenoff R. Sarcopenia: a major modifiable cause of frailty in the elderly. J Nutr Health Aging. 2000;4(3):140-142.

11. Visvanathan R, Chapman I. Preventing sarcopenia in older people. Maturitas. 2010;66(4):383-388. doi:10.1016/j.maturitas.2010.03. 020

12. Keller K. Sarcopenia. Wiener Medizinische Wochenschrift. 2019;169 (7-8):157-172. doi:10.1007/s10354-018-0618-2

13. Studenski SA, Peters KW, Alley DE, et al. The FNIH sarcopenia project: rationale, study description, conference recommendations, and final estimates. J Gerontol Series A. 2014;69(5):547-558. doi:10.1093/gerona/glu010

14. Cruz-Jentoft AJ, Bahat G, Bauer J, et al. Sarcopenia: revised European consensus on definition and diagnosis. Age Ageing. 2019;48(1):16-31. doi:10.1093/ageing/afy169

15. Chen L-K, Woo J, Assantachai P, et al. Asian Working Group for Sarcopenia: 2019 consensus update on sarcopenia diagnosis and treatment. J Am Med Dir Assoc. 2020;21(3):300-307. e302. doi:10.1016/j.jamda.2019.12.012

16. Malmstrom TK, Morley JE. SARC-F: a simple questionnaire to rapidly diagnose sarcopenia. $J$ Am Med Dir Assoc. 2013;14 (8):531-532. doi:10.1016/j.jamda.2013.05.018

17. Kim S, Kim M, Won CW. Validation of the Korean version of the SARC-F questionnaire to assess sarcopenia: Korean frailty and aging cohort study. J Am Med Dir Assoc. 2018;19(1):40-45. e41. doi:10.1016/j.jamda.2017.07.006

18. Bahat G, Yilmaz O, Kilic C, Oren M, Karan M. Performance of SARC-F in regard to sarcopenia definitions, muscle mass and functional measures. $J$ Nutr Health Aging. 2018;22(8):898-903. doi:10.1007/s12603-018-1067-8

19. Woo J, Leung J, Morley JE. Validating the SARC-F: A suitable community screening tool for sarcopenia? J Am Med Dir Assoc. 2014;15(9):630-634. doi:10.1016/j.jamda.2014.04.021

20. Charlson M, Szatrowski TP, Peterson J, Gold J. Validation of a combined comorbidity index. J Clin Epidemiol. 1994;47 (11):1245-1251. doi:10.1016/0895-4356(94)90129-5

21. Tavakol M, Dennick R. Making sense of Cronbach's alpha. Int j Med Educ. 2011;2:53. doi:10.5116/ijme.4dfb.8dfd

22. Linden A. Measuring diagnostic and predictive accuracy in disease management: an introduction to receiver operating characteristic (ROC) analysis. J Eval Clin Pract. 2006;12(2):132-139. doi:10.11 11/j.1365-2753.2005.00598.x

23. Šimundić A-M: Measures of diagnostic accuracy: basic definitions. Ejifcc. 2009;19(4):203.

24. Dent E, Morley J, Cruz-Jentoft A, et al. International clinical practice guidelines for sarcopenia (ICFSR): screening, diagnosis and management. J Nutr Health Aging. 2018;22(10):1148-1161. doi:10. 1007/s12603-018-1139-9

25. Malmstrom TK, Miller DK, Simonsick EM, Ferrucci L, Morley JE. SARC-F: a symptom score to predict persons with sarcopenia at risk for poor functional outcomes. $J$ Cachexia Sarcopenia Muscle. 2016;7(1):28-36. doi:10.1002/jcsm.12 048

26. Parra-Rodriguez L, Szlejf C, Garcia-Gonzalez AI, Malmstrom TK, Cruz-Arenas E, Rosas-Carrasco O. Cross-cultural adaptation and validation of the Spanish-language version of the SARC-F to assess sarcopenia in Mexican community-dwelling older adults. $J$ Am Med Dir Assoc. 2016;17(12):1142-1146. doi:10.1016/j.jamda.2016.09. 008

27. Ida S, Murata K, Nakadachi D, et al. Development of a Japanese version of the SARC-F for diabetic patients: an examination of reliability and validity. Aging Clin Exp Res. 2017;29(5):935-942. doi:10.1007/s40520-016-0668-5

28. Kera T, Kawai H, Hirano H, et al. SARC-F: A validation study with community-dwelling older Japanese adults. Geriatr Gerontol Int. 2019;19(11):1172-1178. doi:10.1111/ggi.13768 
29. Brenner H, Gefeller O. Variation of sensitivity, specificity, likelihood ratios and predictive values with disease prevalence. Stat Med. 1997;16 (9):981-991. doi:10.1002/(SICI)1097-0258(19970515)16:9<981::AIDSIM510>3.0.CO;2-N
30. Artaud F, Singh-Manoux A, Dugravot A, Tzourio C, Elbaz A. Decline in fast gait speed as a predictor of disability in older adults. $J$ Am Geriatr Soc. 2015;63(6):1129-1136. doi:10.1111/jgs.13442

\section{Publish your work in this journal}

Clinical Interventions in Aging is an international, peer-reviewed journal focusing on evidence-based reports on the value or lack thereof of treatments intended to prevent or delay the onset of maladaptive correlates of aging in human beings. This journal is indexed on PubMed Central, MedLine, CAS, Scopus and the Elsevier
Bibliographic databases. The manuscript management system is completely online and includes a very quick and fair peer-review system, which is all easy to use. Visit http://www.dovepress.com/ testimonials.php to read real quotes from published authors. 